(173)

\title{
Antibacterial Activity of Isolated Cyanobacteria Species Oscillatoria sp.
}

\author{
W.A.M.A Wijesekara, P.M. Manage* \\ Department of Zoology, University of Sri Jayawardenepura, Sri Lanka \\ *pathmalal@sjp.ac.lk
}

\begin{abstract}
Cyanobacteria are a highly diverse group of prokaryotes recognised as a potent source of biologically active compounds with antiviral, antibacterial, antifungal, and anticancer properties. In the present study cyanobacterium Oscillatoria sp. was isolated from Senanayaka Samudra Reservoir in Sri Lanka and pure monocultures and mass cultures were prepared. Oscillatoria sp. was harvested after 24 days of incubation at exponential growth to extract compounds using methanol and hexane. Gas chromatography-mass spectrometry (GC-MS) was used to identify compounds present in the crude extract. Antibacterial activity of crude extract was determined by agar well diffusion and disk diffusion methods against gram positive bacteria of Methicillin-resistant Staphylococcus aureus (MRSA) ATCCm25923 and gram negative bacteria of Pseudomonas aeruginosa ATCC 25853, Salmonella typhii and Escherichia coli ATCC 25922. The results showed that the solvents with different polarities had effects on dry biomass of extracted residue. 10\% biomass was extracted by hexane extraction protocol where $60 \%$ of biomass was extracted by methanol extraction. The highest antibacterial activity in methanol extract was detected against S.typhii in disk diffusion method and the mean diameter of inhibition zone was around $22 \mathrm{~mm}$ and mean diameter of inhibition zones of $S$. aureus, P. aeruginosa and E. coli were recorded as 17, 17 and $15 \mathrm{~mm}$ respectively within 24 hours. In agar well diffusion method, methanol extract showed the highest antibacterial activity against $S$. typhii and mean diameter of inhibition was around $54 \mathrm{~mm}$ and mean diameter of inhibition zones of $S$. aureus, $P$. aeruginosa and E. coli were recorded as 52, 50 and $52 \mathrm{~mm}$ respectively within 24 hours. In the hexane extract, antibacterial activities was detected only for $S$. aureus in both methods and mean inhibition zones were recorded in 22 and $11 \mathrm{~mm}$ for agar well diffusion method and disk diffusion method respectively within 24 hours. GC-MS analysis revealed that the crude extract of Oscillatoria contains important fatty acid compounds such as hexadecanoic acid (palmitic acid methyl ester), methyl tetradecaoate (myristic acid methyl ester) and 13Tetradecanoic acid (methyl ester) which possesses antibacterial and antifungal properties. Thus, the result of the study showed that the Osillatoria contained compounds which are potential for pharmaceutical invention.
\end{abstract}

Keywords: Oscillatoria sp. Antibacterial compounds, Well diffusion, Disk diffusion 